\title{
DISTRIBUIÇÃO DE NaCI NO QUEIJO REINO AO LONGO DA MATURAÇÃO
}

\section{Sodium chloride distribution in Reino cheese along the ripening}

\author{
Lia Barbosa Taveira ${ }^{I}$, Renata Golin Bueno Costa ${ }^{1 *}$, Luiz Carlos Gonçalves Costa Júnior ${ }^{I}$, \\ Denise Sobral ${ }^{1}$, Junio César Jacinto de Paula ${ }^{1}$
}

\begin{abstract}
RESUMO
O objetivo foi determinar o tempo mínimo necessário para a distribuição igualitária do sal em todas as partes do queijo Reino, ou seja, desde a superfície até o centro, e verificar se houve diferença significativa nessa distribuição em diferentes condições de maturação. Os queijos de um mesmo lote foram submetidos a dois tratamentos, com diferentes temperaturas, umidade relativa do ar e também com e sem embalagem. No primeiro tratamento o queijo foi maturado sem embalagem termoencolhível, à temperatura de $18{ }^{\circ} \mathrm{C}$ a $22{ }^{\circ} \mathrm{C}$, com $55 \%-65 \%$ de URA. Já no segundo tratamento, o queijo inicialmente foi maturado sem embalagem (apenas nos cinco primeiros dias) e nas mesmas condições do primeiro tratamento e, após este período, foi embalado em película termoencolhível e maturado a $5{ }^{\circ} \mathrm{C}-10{ }^{\circ} \mathrm{C}$, com $70 \%$ - 80\% de URA, até completar o tempo de maturação total estipulado. A caracterização do perfil de distribuição de sal nos queijos Reino foi conduzida por meio da determinação do teor percentual de cloreto de sódio $(\mathrm{m} / \mathrm{m})$ nas porções centrais, intermediárias e superficiais. A análise de variância indicou que não houve diferença entre os tratamentos na distribuição de sal no queijo Reino, porém, houve diferença entre a porção de queijo analisada e o tempo de maturação, o que demonstrou a dependência da distribuição do sal em função destas variáveis. Concluise que a partir do $27^{\circ}$ dia após a fabricação, o sal se distribui homogeneamente em todas as partes do queijo Reino, independentemente das diferentes condições empregadas na maturação.
\end{abstract}

Palavras-chave: embalagem; salga; difusão.

1 Empresa de Pesquisa Agropecuária de Minas Gerais (EPAMIG), Rua Tenente Freitas, 116, Santa Terezinha, 36045-560, Juiz de Fora, MG, Brasil. E-mail: renata.costa@epamig.br

* Autor para correspondência. 


\begin{abstract}
The aim was to determine the minimum time necessary for equal distribution of salt in all parts of Reino cheese, from surface to center, and check if there was significant difference in this distribution in different ripening conditions. The cheeses of the same lot were subjected to two treatments, with different temperatures, relative humidity and with and without packaging. In the first treatment the cheese was ripened without heat-shrinkable packaging at a temperature of $18{ }^{\circ} \mathrm{C}$ to $22^{\circ} \mathrm{C}$ with $55 \%-65 \%$ relative humidity. In the second treatment, the cheese was initially ripened without packaging (only the first five days) and under the same conditions as the first treatment and after this period was packaged in heat-shrinkable packaging and ripening at $5{ }^{\circ} \mathrm{C}-10{ }^{\circ} \mathrm{C}$ with $70 \%-80 \%$ relative humidity, until completing all the prescribed time maturation. The characterization of the salt distribution profile in Reino cheese was conducted by determining the percentage content of sodium chloride $(\mathrm{w} / \mathrm{w})$ in the central, intermediate and superficial portions. Analysis of variance indicated that there was no difference between treatments in the distribution of salt in Reino cheese, however, there were differences between cheese portion analyzed and maturation time, which showed dependence of the distribution of salt depending on these variables. It follows that from the 27 th day after manufacturing, salt is distributed equally in all parts of the Reino cheese, regardless of different ripening conditions used.
\end{abstract}

Keywords: packaging; salting; diffusion.

\section{INTRODUÇÃO}

O Reino é considerado um queijo brasileiro tradicional, originário do Edam holandês. No final do século XIX, era trazido ao Brasil importado do Reino de Portugal e foi o primeiro queijo maturado a ser produzido industrialmente no Brasil, fato de importância histórica para o desenvolvimento da indústria queijeira nacional (FURTADO, 2008).

O queijo Reino é caracterizado por sua textura interna relativamente dura, alaranjada, fechada ou com pequenas olhaduras, casca fina, lisa, de coloração vermelho ou róseo, e, por seu sabor picante e aroma acentuado. Outra característica tradicional desse queijo é sua embalagem em latas esféricas, hermeticamente fechadas, que permitem a manutenção das características típicas do produto (FURTADO, 2007). Durante sua fabricação, é salgado em salmoura entre 36 e 48 horas, com uma concentração de $20 \%$ - 22\% (m/v) de $\mathrm{NaCl}$, a uma temperatura de $10^{\circ} \mathrm{C}$ $12{ }^{\circ} \mathrm{C}$ (FURTADO, 2007; FURTADO, 2008). Durante esse processo de salga, a casca do queijo funciona como membrana semipermeável que permite a ocorrência de dois fluxos importantes: a entrada de $\mathrm{NaCl}$ por difusão e a perda de água e de alguns sólidos solúveis (ácido láctico, lactose, compostos nitrogenados solúveis em TCA a 12\%) presentes no queijo para a salmoura por diferença de pressão osmótica entre a água do queijo e a da salmoura (GUINEE; FOX, 2004; HOFMEISTER et. al, 2005).

A absorção de sal pelo queijo durante a salga sofre influência de alguns fatores como viscosidade da fase aquosa e quantidade de água ligada no queijo, porosidade da massa do queijo e interação da matriz protéica com o sódio (MELLILI et al., 2005). Outros fatores que também influenciam são: concentração, temperatura, $\mathrm{pH}$ e teor de cálcio da salmoura, além dos teores de umidade e gordura, 
tamanho, formato e tempo de salga do queijo (GUINEE, 2004; McSWEENEY, 2007).

$\mathrm{O}$ processo de salga em salmoura geralmente é lento, requer horas ou dias. Ao fim desse período, o queijo é retirado da salmoura e apresenta-se com um gradiente decrescente de sal da superfície para o centro e um gradiente de umidade em direção contrária. Esses gradientes desaparecem lentamente devido à baixa difusão do sal. A condição de equilíbrio, no qual o sal se encontra distribuído homogeneamente no queijo, é atingida durante o período de maturação (FONTAN et al., 2004; GUINEE, 2004). Tanto a fabricação quanto a maturação são influenciadas pelo transporte de solutos aquosos e de água dentro do queijo (FLOURY et al. 2012). O $\mathrm{NaCl}$ e a umidade têm papel importante nas reações bioquímicas, microbiológicas e na qualidade sensorial do queijo (GUINEE; FOX, 2004), e, por este motivo, devem ser temas de pesquisas.

Esse estudo teve como objetivo determinar o tempo necessário para que o sal se distribuísse homogeneamente no queijo Reino e, verificar se houve diferença significativa nessa distribuição quando o mesmo foi submetido a diferentes condições de maturação.

\section{MATERIAL E MÉTODOS}

A fabricação e a estocagem dos queijos Reino foram realizadas em uma fábrica de laticínios, no município de Lima Duarte, Minas Gerais, Brasil.

Três lotes de queijo Reino foram produzidos em escala industrial, sendo que cada lote foi fabricado a partir de 10.000 litros (L) de leite pasteurizado pelo processo HTST $\left(72{ }^{\circ} \mathrm{C} / 15 \mathrm{~s}\right)$ e padronizado para $3,0 \%-3,1 \%$ $(\mathrm{m} / \mathrm{m})$ de teor de gordura, oriundos de um mesmo tanque de estocagem sob refrigeração. Após a pasteurização, o leite foi resfriado a $32{ }^{\circ} \mathrm{C}-34{ }^{\circ} \mathrm{C}$ e adicionado de cloreto de cálcio (solução a $50 \%(\mathrm{~m} / \mathrm{v})$ ) na proporção
$0,40 \mathrm{~mL} / \mathrm{L}$ leite, nitrato de sódio (solução a $50 \%(\mathrm{~m} / \mathrm{v}))$ na proporção $0,40 \mathrm{~mL} / \mathrm{L}$ leite, corante natural vegetal a base de urucum $(0,12$ $\mathrm{mL} / \mathrm{L}$ leite), seguida pela adição de fermento liofilizado na proporção de: $0,8 \%$ de fermento mesofílico (Lactococcus lactis subsp. lactis e Lactococcus lactis subsp. cremoris, Chr. Hansen ${ }^{\mathrm{TM}}$, Brazil), 0,2\% de fermento mesofílico aromático (Lactococcus lactis subsp. cremoris, Lactococcus lactis subsp. lactis, Lactococcus lactis subsp. lactis biovar diacetylactis e Leuconostoc mesenteroides subsp. cremoris, Chr. Hansen ${ }^{\mathrm{TM}}$, Brazil), $0,5 \%$ de fermento termofílico (Lactobacillus helveticus, Chr. Hansen ${ }^{\mathrm{TM}}$, Brazil). A pré-maturação do fermento ocorreu por 30 minutos a $32{ }^{\circ} \mathrm{C}-34{ }^{\circ} \mathrm{C}$. O coagulante líquido contendo $100 \%$ de quimosina (Chr. Hansen ${ }^{\mathrm{TM}}$, Brazil) foi adicionado na dosagem recomendada pelo fabricante.

A coagulação ocorreu em $35 \pm 5$ minutos. A coalhada foi cortada em cubos de aproximadamente $0,3 \mathrm{~cm}-0,5 \mathrm{~cm}$, seguido de mexedura lenta por 15 minutos. Para o processo de aquecimento, $30 \%$ de soro (v/v) foi removido em relação ao volume de leite, e reposto progressivamente com adição de $20 \%(\mathrm{v} / \mathrm{v})$ de água quente $\left(75^{\circ} \mathrm{C}-80^{\circ} \mathrm{C}\right)$ a coalhada, sob agitação contínua, para aumentar a temperatura de $1{ }^{\circ} \mathrm{C}$ a cada 2 minutos, até a temperatura de $45^{\circ} \mathrm{C}$. No tempo de 70 a 90 minutos após o corte, o soro foi drenado, a coalhada foi pré-prensada por 20 minutos a $0,065 \mathrm{MPa}$, enformada e prensada em formas esféricas de $1,5 \mathrm{~kg}$, por 20 horas com 0,4 MPa.

Os queijos foram salgados em salmoura $(23 \% \mathrm{NaCl}(\mathrm{m} / \mathrm{m}))$ por aproximadamente 24 horas, secados por 10 dias a $14,3{ }^{\circ} \mathrm{C}$ e $96 \%$ URA e pintados com tintura a base de fucsina e outros compostos para proteção contra fungos. A tecnologia de elaboração do queijo Reino foi padronizada para obter queijo com massa de um quilograma, em média, e $13 \mathrm{~cm}$ de diâmetro.

Os queijos Reino do mesmo lote de fabricação foram divididos em dois tratamentos 
(A e B) e analisados a partir do primeiro dia de maturação até o final dessa etapa (25 dias). No tratamento A, os queijos foram maturados sem embalagem à temperatura entre $18{ }^{\circ} \mathrm{C} \mathrm{e}$ $22{ }^{\circ} \mathrm{C}$, com $55 \%$ - $65 \%$ de URA por 25 dias. Já no tratamento B, os queijos foram maturados por cinco dias nas condições do tratamento A $\left(18{ }^{\circ} \mathrm{C}\right.$ a $22{ }^{\circ} \mathrm{C}$, com 55\% - $65 \%$ de URA), e depois foram embalados em película termoencolhível e mantidos por mais 20 dias em câmara entre $5{ }^{\circ} \mathrm{C}$ e $10{ }^{\circ} \mathrm{C}$, com $70 \%$ - 80\% de URA, perfazendo ao final, os mesmos 25 dias de maturação.

Os queijos foram analisados durante a maturação nos tempos de 1, 6, 15 e 25 dias. Fez-se a análise dos teores percentuais $(\mathrm{m} / \mathrm{m})$ de cloretos $(\mathrm{NaCl})$, sólidos totais e de gordura (BRASIL, 2006). Posteriormente, foram calculados os teores percentuais $(\mathrm{m} / \mathrm{m})$ de gordura no extrato seco ( $\%$ GES) e de sal na umidade dos queijos ( $\% \mathrm{~S} / \mathrm{U})$.

A caracterização do perfil de distribuição de $\mathrm{NaCl}$ nos queijos Reino foi conduzida por meio da determinação do teor percentual de cloretos $(\mathrm{m} / \mathrm{m})$, a partir da retirada de uma amostra na parte central, perfurando de um lado ao outro com emprego de sonda de aço inoxidável $(30 \mathrm{~cm}$ de comprimento e 1,5 $\mathrm{cm}$ de diâmetro). Essa amostra foi dividida em seis partes iguais (Figura 1), de modo a obter duas porções centrais (C), duas intermediárias (I) e duas superficiais (S), que foram analisadas separadamente para verificação da concentração de $\mathrm{NaCl}$ (sal) em cada porção individual (BRASIL, 2006). O percentual de $\mathrm{NaCl}$ dos queijos foi representado pela média dos resultados obtidos em cada uma das três porções ( C, I e $\mathrm{S})$, até que os resultados analíticos das mesmas se equiparassem, de forma a comprovar que naquele tempo o $\mathrm{NaCl}$ já estava distribuído homogeneamente no queijo.

Ao todo, o queijo demorou 37 dias para ficar pronto, sendo os 12 primeiros dias contabilizados para salga e secagem do queijo e os 25 dias restantes, contabilizados como o período de maturação. Para avaliar a difusão do $\mathrm{NaCl}$, os queijos foram analisados nos dias 2, 7, 12, 17, 27 e 37 dias após a fabricação.

Empregou-se o delineamento em parcelas subdivididas, com três repetições. As definições de parcelas e subparcelas em cada etapa experimental estão descritas na Tabela 1.
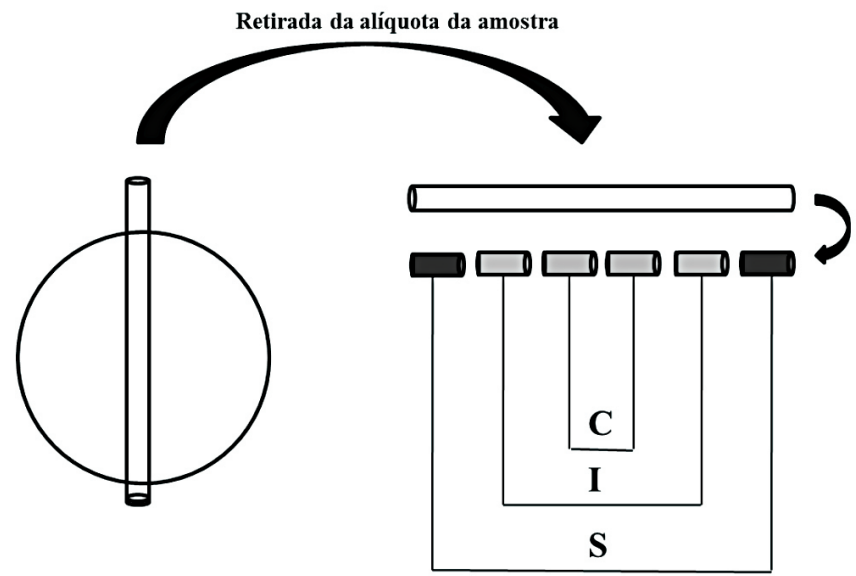

Fracionamento da alíquota nas porções S, I e C.

Figura 1 - Representação da metodologia utilizada para retirar a alíquota da amostra por sondagem, a qual atravessou todo o perfil e onde "C" representa as 2 porções centrais, "I" as 2 intermediárias e " $\mathrm{S}$ " as 2 superficiais do queijo Reino 
Os resultados do experimento foram submetidos ao Teste de Shapiro-Wilk, para verificar a normalidade dos resíduos, como pressuposto à análise de variância paramétrica. Foi, então, realizada a análise de variância e o Teste de Student Newman-Keuls (SNK) em nível de 5\% de probabilidade por meio do programa SISVAR (FERREIRA, 2011).

\section{RESULTADOS E DISCUSSÃO}

O teor de extrato seco total do queijo Reino aumentou ao longo da maturação apenas no tratamento $\mathrm{A}(\mathrm{p}<0,05)$ (Tabela 2$)$, devido às condições de maturação submetidas nesse tratamento, como URA mais baixa (55\% - 65\%) e maturação sem embalagem. No tratamento B, o uso da embalagem em película termoencolhível juntamente com o teor de URA da câmara de maturação mais elevado (passando de 55\% - 65\% para 70\% - 80\%) impediu a perda de umidade por desidratação e manteve o teor de sólidos totais constante. O controle do teor de umidade do queijo durante a maturação pode ser realizado pelo aumento da umidade relativa do ar da câmara de maturação ou pela embalagem do queijo (BERESFORD et al, 2001).

No entanto, não houve diferença significativa $(\mathrm{p}>0,05)$ no teor de extrato seco total entre os tratamentos A e B. Simal et al. (2001) observaram maior desidratação e maior percentual de sólidos totais em queijos Mahon maturados a $70 \%$ de URA do que naqueles a $80 \%$ de URA, durante 115 dias de maturação. No entanto, no presente trabalho, o tempo de 25 dias de maturação não foi suficiente para provocar essa diferença na umidade do queijo, possivelmente devido ao curto período de maturação. Caso o período de maturação fosse estendido, talvez houvesse diferença significativa entre a umidade entre os tratamentos dos queijos maturados em diferentes condições de umidade relativa.

O teor percentual $(\mathrm{m} / \mathrm{m})$ de gordura no extrato seco (GES) permaneceu constante ao longo do tempo de maturação $(p>0,05)$ para cada tratamento. Os queijos Reinos dos tratamentos A e B podem ser classificados como gordo (GES entre 45,0\% - 59,9\% m/m) segundo as especificações do Regulamento Técnico de Identidade e Qualidade de Queijos (BRASIL, 1996).

Tabela 1 - Definições de parcelas e subparcelas em cada etapa experimental

\begin{tabular}{|c|c|c|c|}
\hline & Parcelas & Subparcelas 1 & $\begin{array}{c}\text { Subparcelas } 2 \\
\text { (tempo após } \\
\text { a fabricação) }\end{array}$ \\
\hline $\begin{array}{l}\text { Análises físico-químicas } \\
\text { do queijo Reino } \\
\text { nos tempos } 1,6,15 \text { e } 25 \\
\text { dias de maturação }\end{array}$ & $\begin{array}{l}\text { Tratamento A } \\
\text { Tratamento B }\end{array}$ & $\begin{array}{c}\text { Tempo } 1 \\
\text { Tempo } 6 \\
\text { Tempo } 15 \\
\text { Tempo } 25\end{array}$ & \\
\hline $\begin{array}{l}\text { Distribuição de } \mathrm{NaCl} \\
\text { no queijo Reino }\end{array}$ & $\begin{array}{l}\text { Tratamento A } \\
\text { Tratamento B }\end{array}$ & $\begin{array}{c}\text { Superficial A } \\
\text { Intermediária A } \\
\text { Central A } \\
\text { Superficial B } \\
\text { Intermediária B } \\
\text { Central B }\end{array}$ & $\begin{array}{c}\text { Tempo } 2 \\
\text { Tempo } 7 * \\
\text { Tempo } 12 * \\
\text { Tempo } 17 * * \\
\text { Tempo } 27 * * \\
\text { Tempo } 37 * *\end{array}$ \\
\hline
\end{tabular}

* corresponde aos tempos 5 e 10 dias de secagem; ** corresponde aos tempos 6,15 e 25 dias de maturação 
$\mathrm{O}$ queijo do tratamento $\mathrm{A}$ apresentou menor teor de GES aos 25 dias em comparação ao tratamento B $(\mathrm{p}<0,05)$, por ter sido maturado em temperatura mais elevada, com URA mais baixa e sem embalagem, apesar da tendência do GES se manter constante durante a maturação na maioria dos queijos. Embora não tenha havido diferença significativa no extrato seco no queijo do tratamento $\mathrm{A}$, no cálculo do GES esse valor numericamente menor influenciou o resultado.

O teor percentual $(\mathrm{m} / \mathrm{m})$ de sal na umidade $(\mathrm{S} / \mathrm{U})$ do queijo aumentou ao longo da maturação $(\mathrm{p}<0,05)$. Este comportamento demonstrou que o $\mathrm{NaCl}$ se concentrou no queijo em função da desidratação decorrente do processo de maturação. Porém, a aplicação dos tratamentos A e B nos queijos provocaram efeitos diferentes sobre a variação do \% de $\mathrm{S} / \mathrm{U}$ ao longo do tempo. Os queijos do tratamento A apresentaram maior variação que os do $\mathrm{B}$, devido às diferentes condições durante $\mathrm{a}$ maturação, o que pode ser verificado também no aumento do teor percentual de extrato seco total. Os queijos do tratamento B, aos cinco dias de maturação, foram embalados e após esse período não houve diferença $(\mathrm{p}>0,05)$ no teor de $\mathrm{S} / \mathrm{U}$ entre os tempos 6,15 e 25 dias de maturação. Como o teor de extrato seco também não variou nesse período, consequentemente, a umidade também não foi alterada e por isso, o teor de $\mathrm{S} / \mathrm{U}$ se manteve estatisticamente constante no tratamento B ao longo da maturação.

Em relação à difusão do $\mathrm{NaCl}$ dentro do queijo não houve diferença significativa entre os tratamentos $(\mathrm{p}>0,05)$, ou seja, as condições de maturação não influenciaram na difusão do sal. Embora o tempo de equilíbrio do teor de $\mathrm{NaCl}$ dependa do tipo de queijo, composição, forma e tamanho e ainda, das condições de maturação (GUINEE, 2004), nesse trabalho estas diferenças não influenciaram na difusão.

Os queijos dos tratamentos A e B passaram pelo mesmo processo de salga, secagem e início da maturação (os primeiros cinco dias de maturação foram iguais para ambos os tratamentos). Este período inicial de 17 dias pode ter contribuído para o comportamento semelhante de distribuição do sal dos tratamentos A e B, sem diferença

Tabela 2 - Teores percentuais $(\mathrm{m} / \mathrm{m})$ de extrato seco total (EST), gordura no extrato seco (GES) e sal na umidade $(\mathrm{S} / \mathrm{U})$ dos queijos Reino durante a maturação* $(\mathrm{n}=3)$

\begin{tabular}{|c|c|c|c|c|c|}
\hline & \multirow{2}{*}{ Tratamentos** } & \multicolumn{4}{|c|}{ Tempo de maturação (dias) } \\
\hline & & 1 & 6 & 15 & 25 \\
\hline EST & A & $61,58 \pm 2,08^{\mathrm{aA}}$ & $62,77 \pm 0,20^{\mathrm{aAB}}$ & $65,32 \pm 1,13^{\mathrm{aBC}}$ & $66,40 \pm 1,81^{\mathrm{aC}}$ \\
\hline$(\% \mathrm{~m} / \mathrm{m})$ & $\mathrm{B}$ & $62,04 \pm 2,71^{\mathrm{aA}}$ & $62,71 \pm 0,24^{\mathrm{aA}}$ & $64,18 \pm 2,09^{\mathrm{aA}}$ & $65,16 \pm 1,56^{\mathrm{aA}}$ \\
\hline GES & A & $51,15 \pm 1,45^{\mathrm{aA}}$ & $50,98 \pm 1,46^{\mathrm{aA}}$ & $48,44 \pm 2,46^{\mathrm{aA}}$ & $47,07 \pm 2,81^{\mathrm{bA}}$ \\
\hline$(\% \mathrm{~m} / \mathrm{m})$ & $\mathrm{B}$ & $51,77 \pm 0,64^{\mathrm{aA}}$ & $50,22 \pm 0,93^{\mathrm{aA}}$ & $50,23 \pm 1,30^{\mathrm{aA}}$ & $52,07 \pm 1,20^{\mathrm{aA}}$ \\
\hline $\mathrm{S} / \mathrm{U}$ & A & $2,29 \pm 0,44^{\mathrm{aC}}$ & $3,09 \pm 0,11^{\mathrm{aB}}$ & $4,07 \pm 0,23^{\mathrm{aA}}$ & $4,27 \pm 0,76^{\mathrm{aA}}$ \\
\hline$(\% \mathrm{~m} / \mathrm{m})$ & B & $2,44 \pm 0,49^{\mathrm{aB}}$ & $3,14 \pm 0,09^{\mathrm{aA}}$ & $3,80 \pm 0,10^{\mathrm{aA}}$ & $3,86 \pm 0,80^{\mathrm{aA}}$ \\
\hline \multicolumn{6}{|c|}{$\begin{array}{l}\text { * Letras iguais indicam valores semelhantes pelo Teste de Student Newman-Keuls (SNK) em nível de } 5 \% \\
\text { de probabilidade. Letras maiúsculas devem ser consideradas entre os tempos de cada tratamento (linha) e, } \\
\text { minúsculas entre os Tratamentos A e B (coluna). **Tratamento A - maturação sem embalagem à temperatura } \\
\text { entre } 18^{\circ} \mathrm{C} \text { e } 22{ }^{\circ} \mathrm{C} \text {, com } 55 \% \text { - } 65 \% \text { de URA. Tratamento B - maturação por cinco dias nas condições do } \\
\text { tratamento A, e depois embalagem em película termoencolhível e maturação em câmara fria }\left(5{ }^{\circ} \mathrm{C}-10^{\circ} \mathrm{C}\right) \\
\text { com } 70 \%-80 \% \text { de URA }\end{array}$} \\
\hline
\end{tabular}


significativa. Sendo assim, a distribuição do $\mathrm{NaCl}$ nos queijos tornou-se igualitária em todas as partes do queijos aos 15 dias de maturação ( $27^{\circ}$ dia após fabricação) (Figura 2) para ambos os tratamentos, retratando que possivelmente as alterações nas condições de maturação tiveram pouca influência na distribuição do sal nos dois tratamentos.

Quanto à difusão do $\mathrm{NaCl}$ dentro do queijo, houve diferença entre as porções amostradas do queijo Reino $(\mathrm{p}<0,05)$ e ao longo do tempo $(\mathrm{p}<0,05)$. Logo após o processo de salga (dois dias após a fabricação), o sal se encontrou concentrado na porção superficial do queijo, e, com o decorrer do tempo, se difundiu para o interior do mesmo (Figura 2). No processo de salga em salmoura, a superfície do queijo entra em contato direto com a salmoura, uma solução concentrada de sal, absorve sal ao mesmo tempo em que perde umidade, o que leva à desidratação e a formação da casca do queijo.

Simal et al. (2001) também observaram no queijo Mahon que a concentração de $\mathrm{NaCl}$ apresentou uma grande variação do centro até a superfície do queijo no início da maturação. Desde o início da maturação, no presente estudo, percebe-se um declínio no teor de sal da região superficial do queijo, com consequente aumento nas regiões central e intermediária. Após a retirada do queijo da salmoura, o sal se difunde para o interior do queijo para que ocorra uma distribuição homogênea em todo o queijo. Esta difusão de sal durante o período de maturação é semelhante a de outros trabalhos (GEURTS et al.,1974; GUINEE; FOX,1985; PRASAD; ALVAREZ,1999; PONCE DE LEONGONZALEZ et al., 2000, Costa et al., 2005; MUCCHETTI; NEVIANI, 2006). Prasad; Alvarez (1999) verificaram um aumento maior no teor de $\mathrm{NaCl}$ no interior do queijo Feta na primeira semana de maturação. Costa et al. (2005) também verificaram uma maior redução do teor de $\mathrm{NaCl}$ da casca do queijo Prato após 10 dias de maturação com um aumento do teor de $\mathrm{NaCl}$ no interior do queijo. Outros tipos de salga também apresentam o mesmo mecanismo de difusão do sal, como a salga a seco. Santapaola et al. (2013) verificaram um gradiente de sal da superfície para o interior em queijo de cabra salgado a seco.

A distribuição de $\mathrm{NaCl}$ depende do tipo de queijo (tamanho e formato), mas principalmente do teor de umidade e de $\mathrm{NaCl}$. Em queijo Prato de 0,5 kg maturado embalado,

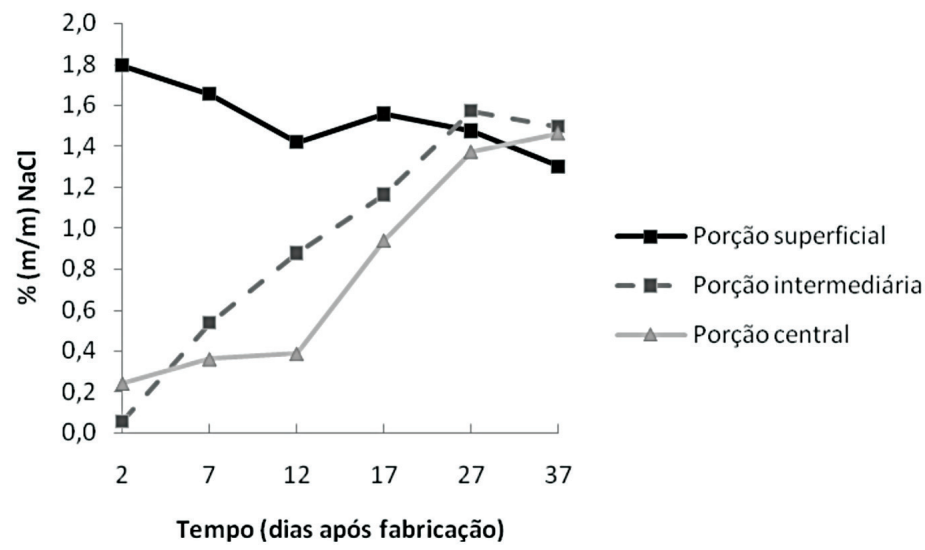

Figura 2 - Evolução da média de distribuição de sal $(\%(\mathrm{~m} / \mathrm{m})$ de $\mathrm{NaCl})$ nas porções do queijo Reino, dos dois tratamentos, desde a saída da salga (com 2 dias), secagem (até 12 dias) e final da maturação (37 dias) 
com 42,1\% $(\mathrm{m} / \mathrm{m})$ de umidade, o teor de $\mathrm{NaCl}$ não se igualou entre a casca e o interior do queijo após 20 dias de maturação (COSTA et al., 2005). Para o queijo Edam esférico de $2,5 \mathrm{~kg}$ com teor de umidade de $46 \%(\mathrm{~m} / \mathrm{m})$ o tempo necessário para que o teor de sal esteja uniforme é de 4 a 6 semanas (GUINNE; SUTHERLAND, 2011), o que é altamente dependente das condições de maturação, aliado à composição dos queijos, principalmente, os teores de $\mathrm{NaCl}$ e umidade.

Embora, nos primeiros 12 dias após a fabricação já houvesse redução do teor de $\mathrm{NaCl}$ entre as frações superficiais do queijo Reino analisadas e o sal auxilie no sabor, é necessária a maturação por um período maior para que o sal se distribua de maneira uniforme. Durante a maturação, devido aos princípios de transporte molecular, o queijo alcança um perfil de concentração de sal uniforme (GOMES et al., 1998; GUINEE, 2004). Por isso, neste estudo, comprovouse que a partir do $27^{\circ}$ dia após a fabricação (aos 15 dias de maturação), o teor de $\mathrm{NaCl}$ se igualou no queijo para os tratamentos $\mathrm{A}$ e B $(p>0,05)$. Neste período, o sal estava distribuído homogeneamente em todas as partes do queijo, independentemente das condições de maturação utilizadas.

\section{CONCLUSÕES}

Independente das condições de temperatura e umidade aplicadas na maturação, o sal se distribui homogeneamente em todas as partes no queijo Reino aos 15 dias de maturação, ou 27 dias após a fabricação. Dessa maneira, as indústrias devem respeitar o tempo necessário para que o queijo adquira o gosto salgado característico e uniforme, a fim de assegurarem sua qualidade sensorial.

\section{AGRADECIMENTOS}

À Fapemig pelo apoio financeiro ao projeto e pela concessão da Bolsa de Desen- volvimento Tecnológico Industrial da primeira autora.

\section{REFERÊNCIAS}

BRASIL. Ministério da Agricultura, Pecuária e Abastecimento. Portaria $n^{\circ} 146$, de 7 de março de 1996. Aprova os regulamentos técnicos de identidade e qualidade dos produtos lácteos. Diário Oficial da República do Brasil, Brasília, 11 mar. 1996. Seção 1, Página 3977.

BRASIL. Ministério da Agricultura, Pecuária e Abastecimento. Instrução Normativa no 68, de 12 de dezembro de 2006. Oficializa os métodos analíticos oficiais físico-químicos, para controle de leite e produtos lácteos. Diário Oficial da República do Brasil, Brasília, 14 dez. 2006. Seção 1, p. 8.

BERESFORD et al. Recents advances in cheese microbiology. International Dairy Journal, v. 11, n. 4-7, p. 259-274, 2001.

COSTA, R. G. B.; LOBATO, V.; ABREU, L. R. Aspectos físico-químicos do queijo Prato submetido a salga em salmoura estática e com agitação. Revista do Instituto de Laticínios Cândido Tostes, v. 60, n. 344, p. 3-16, 2005.

FERREIRA, D. F. Sisvar: A computer statistical analysis system. Ciência e Agrotecnologia, v. 35, n. 6, p. 1039-1042, 2011.

FLOURY, J. et al. First assessment of diffusion coefficients in model cheese by fluorescence recovery after photobleaching (FRAP) Food Chemistry, v. 133, n. 2, p. 551-556, 2012.

FONTAN, R. C. I. et al. Determinação do coeficiente efetivo de difusão do cloreto de sódio em queijo Mussarela. Revista do Instituto de Laticínios Cândido Tostes, v. 59, n. 336-338, p. 7-9, 2004. 
FURTADO, M. R. A. Caracterização histórica, tecnologia de fabricação, características físico-químicas, sensoriais, perfil de textura e de comercialização do queijo Reino. 2008. 95 f. Tese (Doutorado em Ciências dos Alimentos) - Universidade Federal de Lavras, Lavras, 2008.

FURTADO, M. M. Queijo com olhaduras. $1^{\mathrm{a}}$ ed. São Paulo: Fonte Comunicações, 2007. $179 \mathrm{p}$.

FURTADO, M. M. Queijos Duros. $1^{\mathrm{a}}$ ed. São Paulo: Setembro Editora, 2011. 212 p.

GEURTS, T. J.; WALSTRA, P.; MULDER, H. Transport of salt and water during salting of cheese. 1. Analysis of the processes involved. Netherlands Milk Dairy Journal, v. 28, p. 102-129, 1974.

GOMES, A. M. P.; VIEIRA, M. M.; MALCATA, F. X. Survival of probiotic microbial strains in a cheese matrix during ripening: Simulation of rates of salt difusion and microorganism survival. Journal of Food Engineering, v. 36, n. 3, p. 281-301, 1998.

GUINEE, T. P.; FOX, P. F. Transport of sodium chloride and water in Romano cheese slices during brining. Food Chemistry, v. 19, n. 1, p. 49-64, 1985.

GUINEE, T. P. Salting and the role of salt in cheese. International Journal of Dairy Technology, v. 57, n. 2/3, p. 99-109, 2004.

GUINEE, T. P.; FOX, P. F. Salt in cheese: physical, chemical and biological aspects. In: FOX, P. F. Cheese: chemistry, physics and microbiology. $3^{\mathrm{a}}$ ed. London: Chapman \& Hall, 2004. v. 1, p. 207-259.
GUINEE, T. P.; SUTHERLAND, B. J. Salting of cheese In: FUQUAY, J. W.; FOX, P. F.; McSWEENEY, P. L. H. Encyclopedia of Dairy Science. London: Academic Press., 2011. p. 595-606.

HOFMEISTER, L. C. et al. Salga de queijo tipo Minas por impregnação a vácuo. Ciência e Tecnologia de Alimentos, v. 25, n. 3, p. 487-494, 2005.

MUCCHETTI G., NEVIANI E. Microbiologia e tecnologia lattierocasearia. Qualità e sicurezza. Milan, Italy: Tecniche Nuove, 2006. 813 p.

McSWEENEY, P. L. H. Cheese problems solved. Woodhead Publishing Limited, England, 2007. 425p.

PONCE DE LEON-GONZALEZ L. et al. Influence of salting procedure on the composition of Muenster-type cheese. Journal of Dairy Science, v. 83, n. 6, p. 1396-1401, 2000.

PRASAD, N.; ALVAREZ, V. B. Effect of salt and chymosin on the physico-chemical properties of Feta cheese during ripening. Journal of Dairy Science, v. 82, n. 6, p. 1061-1067, 1999.

SANTAPAOLA, J.; MALDONADO, S.; MEDINA, J. L. $\mathrm{NaCl}$ diffusion kinetics in dry salting of goat cheese. Journal of Food Engineering, v. 118, n. 2, p.172-177, 2013.

SIMAL, S. et al. Water and salt diffusion during cheese ripening: effect of the external and internal resistances to mass transfer. Journal of Food Engineering, v. 48, n. 3, p. 269-275, 2001 